\title{
A Unified Equation for the Reaction Rate in Dense Matter Stars
}

\author{
L. R. Gasques*, M. Wiescher ${ }^{\dagger}$ and D. G. Yakovlev** \\ *Department of Nuclear Physics, Research School of Physical Sciences and Engineering, \\ Australian National University, Canberra, ACT 0200, Australia \\ ${ }^{\dagger}$ Department of Physics \& The Joint Institute for Nuclear Astrophysics, University of Notre Dame, \\ Notre Dame, IN 46556 USA \\ **Ioffe Physico-Technical Institute, Poliekhnicheskaya 26, 194021 Saint-Petersburg, Russia
}

\begin{abstract}
We analyze thermonuclear and pycnonuclear reaction rates in multi-component dense stellar plasma. First we describe calculations of the astrophysical $S$-factor at low energies using the São Paulo potential on the basis of the barrier penetration model. Then we present a simple phenomenological expression for a reaction rate. The expression contains several fit parameters which we adjust to reproduce the best microscopic calculations available in the literature.
\end{abstract}

Keywords: Astrophysical S-factor; reaction rate in different burning regimes; multi-component plasma stellar matter

PACS: 25.60.Pj,24.10.-i,26.50.+x

\section{INTRODUCTION}

The determination of nuclear fusion rates in dense stellar matter is crucial for understanding the structure and evolution of stars of various types. This problem has two aspects. The first one belongs to nuclear physics and is concerned with the determination of the astrophysical $S$-factor at low energies. In previous works $[1,2,3]$ we demonstrated that the São Paulo (SP) potential model provides a global and reliable description of the fusion processes in the stellar energy range. This model does not contain any free parameter and represents a powerful tool to predict average low energy cross sections for a wide range of fusion reactions. The second issue is related to plasma physics and consists in calculating the Coulomb barrier penetration by reacting nuclei in dense matter taken into account Coulomb fields of surrounding plasma particles. These fields modify the Coulomb barrier resulting in five nuclear burning regimes [4] (two thermonuclear regimes, with weak and strong plasma screening; two pycnonuclear regimes, for zerotemperature and thermally enhanced burning; and the intermediate regime). Recently [3] we have proposed a phenomenological expression to describe the rate in all five regimes for any non-resonant fusion reaction. It generalizes the expression for the reaction rate involving equal nuclei in a one-component plasma (OCP) which we derived earlier [2]. The present expression gives reliable results in the thermonuclear regimes and provides a reasonable description of the Coulomb tunneling problem in other regimes. It can be used in computer codes which simulate a broad range of nuclear burning scenarios, from thermonuclear burning in hot stellar plasma to pycnonuclear burning in high density stellar matter, particularly, in white dwarf cores and neutron star crusts.

CP947, VII Latin American Symposium on Nuclear Physics and Applications edited by R. Alarcon, P. L. Cole, C. Djalali, and F. Umeres (C) 2007 American Institute of Physics 978-0-7354-0461-8/07/\$23.00 


\section{FUSION CROSS SECTION AND ASTROPHYSICAL $S$-FACTOR}

For illustration, we consider nuclear burning in dense stellar carbon-oxygen matter. In this case we need fusion cross sections for the ${ }^{12} \mathrm{C}+{ }^{12} \mathrm{C},{ }^{12} \mathrm{C}+{ }^{16} \mathrm{O}$, and ${ }^{16} \mathrm{O}+{ }^{16} \mathrm{O}$ reactions. In a simple picture, a fusion reaction takes place when colliding nuclei tunnel through the effective barrier potential; thereby their wave functions overlap. A crucial ingredient in the determination of the tunneling probability is the real part of the nuclear potential that describes interaction between two colliding nuclei. To this aim we adopt the SP potential, which is a totally parameter-free model based on nonlocal quantum effects [5]. Within the nonlocal model, the bare interaction $V_{S P}$ is connected with the folding potential $V_{F}$ through

$$
V_{S P}(R, E)=V_{F}(R) \exp \left(-4 v^{2} / c^{2}\right),
$$

where $c$ is the speed of light and $v$ is the local relative velocity of the two nuclei,

$$
v^{2}(R, E)=(2 / \mu)\left[E-V_{C}(R)-V_{S P}(R, E)\right] ;
$$

$V_{C}(r)$ is the Coulomb potential, and $\mu$ is the reduced mass.

According to the double-folding model, the heavy-ion nuclear potential depends on the nuclear densities of the colliding nuclei. Here we adopt the two-parameter Fermi distribution to describe the nuclear densities. The radii of these distributions are well described by the formula [5] $R_{0}=1.31 A^{1 / 3}-0.84 \mathrm{fm}$. The ${ }^{12} \mathrm{C}$ and ${ }^{16} \mathrm{O}$ diffuseness was taken to be $a=0.56 \mathrm{fm}$ and $a=0.58 \mathrm{fm}$, respectively [6,7]. The folding potential was calculated in the zero-range approximation with $V_{0}=-456 \mathrm{MeV} \mathrm{fm}^{3}[5]$.

The effective potential is given by the sum of the Coulomb, nuclear and centrifugal parts:

$$
V_{e f f}(R, E)=V_{C}(R)+V_{S P}(R, E)+\frac{\ell(\ell+1) \hbar^{2}}{2 \mu R^{2}} .
$$

The reaction cross section calculated within the barrier penetration (BP) model is determined by the flux transmitted through the barrier,

$$
\sigma(E)=\frac{\pi}{k^{2}} \sum(2 \ell+1) T_{\ell} ;
$$

the sum is performed up to a maximum $\ell$ wave, corresponding to the greatest angular momentum that produces a pocket. Usually, fusion cross sections $\sigma(E)$ at low energies, typical for astrophysical conditions, are expressed in terms of the astrophysical $S$-factor,

$$
S(E)=\sigma(E) E \exp (2 \pi \eta),
$$

where $\eta=\left(Z_{1} Z_{2} e^{2} / \hbar\right) \sqrt{\mu /(2 E)}$ is the Gamow parameter, $Z_{1}$ and $Z_{2}$ are charge numbers of the nuclei.

The experimentally determined $S$-factors for the ${ }^{12} \mathrm{C}+{ }^{12} \mathrm{C}[8,9,10,11,12,13,14,15$, $16,17,18,19],{ }^{12} \mathrm{C}+{ }^{16} \mathrm{O}[20,21]$ and ${ }^{16} \mathrm{O}+{ }^{16} \mathrm{O}[22,23,24,25,26,27]$ reactions are presented in Fig. 1. In particular, for the ${ }^{12} \mathrm{C}+{ }^{12} \mathrm{C}$ reaction, large discrepancies between the different experimental data at low energies complicate a reliable extrapolation of 

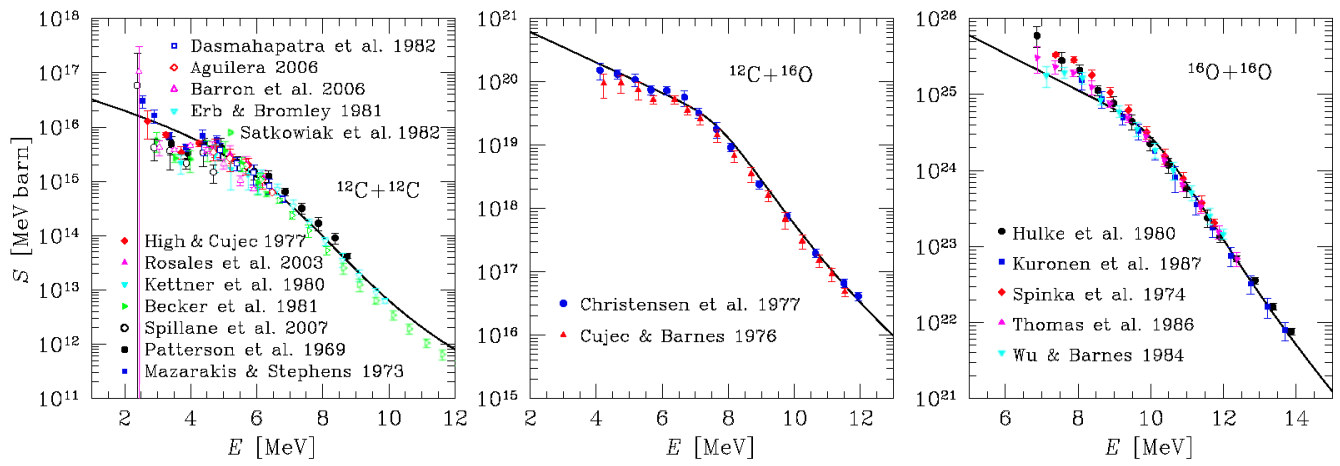

FIGURE 1. (Color online) Astrophysical $S$-factors for the ${ }^{12} \mathrm{C}+{ }^{12} \mathrm{C},{ }^{12} \mathrm{C}+{ }^{16} \mathrm{O}$, and ${ }^{16} \mathrm{O}+{ }^{16} \mathrm{O}$ reactions versus center-of-mass energy $E$ of colliding nuclei (binned over $\Delta E=0.5 \mathrm{MeV}$ intervals). Various symbols represent experimental data. The solid lines show the BP model calculations (see text for details).

TABLE 1. The coefficients $A_{1}, \ldots, A_{5}$ and $E_{0}$ in the fit expression (6) for the $S$-factors of the $\mathrm{CC}, \mathrm{CO}$ and $\mathrm{OO}$ reactions.

\begin{tabular}{ccccccc}
\hline Reaction & $E_{0}$ & $A_{1}$ & $A_{2}$ & $A_{3}$ & $A_{4}$ & $A_{5}$ \\
\hline${ }^{12} \mathrm{C}+{ }^{12} \mathrm{C}$ & 6.946 & 34.75 & -0.552 & -2.131 & -0.625 & 0.0315 \\
${ }^{12} \mathrm{C}+{ }^{16} \mathrm{O}$ & 8.290 & 44.32 & -0.561 & -1.480 & -0.910 & 0.0387 \\
${ }^{16} \mathrm{O}+{ }^{16} \mathrm{O}$ & 10.52 & 56.16 & -0.571 & -1.160 & -1.044 & 0.0366 \\
\hline
\end{tabular}

$S(E)$ towards very low energies. Although resonances exhibited in the ${ }^{12} \mathrm{C}+{ }^{12} \mathrm{C}$ and ${ }^{12} \mathrm{C}+{ }^{16} \mathrm{O}$ data at energies below the fusion barrier cannot be reproduced by the $\mathrm{BP}$ model, this simple model can provide a satisfactory average description of the fusion cross section for the entire energy range. Such a description is sufficient since the reaction rate formalism relies on the $S$-factor averaged over the Gamow-peak range.

The values of $S(E)$ calculated at $E \leq 20 \mathrm{MeV}$ can be fitted by an analytic expression

$$
S(E)=\exp \left(A_{1}+A_{2} \Delta E+\frac{A_{3}+A_{4} \Delta E+A_{5} \Delta E^{2}}{1+\mathrm{e}^{-\Delta E}}\right) \mathrm{MeV} \mathrm{b},
$$

where $\Delta E=E-E_{0}$; the center-of-mass energy $E$ and the fit parameter $E_{0}$ are expressed in megaelectron-volts. Table 1 (from Ref. [3]) gives the fit parameters $A_{1}, \ldots, A_{5}$ and $E_{0}$ for the ${ }^{12} \mathrm{C}+{ }^{12} \mathrm{C},{ }^{12} \mathrm{C}+{ }^{16} \mathrm{O}$ and ${ }^{16} \mathrm{O}+{ }^{16} \mathrm{O}$ reactions. The maximum formal fit errors are $7.2 \%$ at $E=19.8 \mathrm{Mev}$ for $\mathrm{CC} ; 6.3 \%$ at $E=7.5 \mathrm{MeV}$ for CO; and $3.9 \%$ at $E=8.2 \mathrm{MeV}$ for the $\mathrm{OO}$ reaction.

\section{NUCLEAR FUSION RATE IN DENSE STELLAR MATTER}

Now we focus on the plasma physics aspects of nuclear burning in dense matter, where ions are fully ionized and electrons form a uniform background. Let us consider a 
TABLE 2. The parameters $C_{\mathrm{exp}}, C_{\mathrm{pyc}}, C_{\mathrm{pl}}, C_{T}, \alpha_{\lambda}, \alpha_{\omega}$, and $\Lambda$ that define the optimal model and for the models which maximize and minimize the reaction rate.

\begin{tabular}{lccccccc}
\hline Model & $C_{\mathrm{exp}}$ & $C_{\mathrm{pyc}}$ & $C_{\mathrm{pl}}$ & $C_{T}$ & $\alpha_{\lambda}$ & $\alpha_{\omega}$ & $\Lambda$ \\
\hline Optimal & 2.638 & 3.90 & 1.25 & 0.724 & 1 & 1 & 0.5 \\
Maximum rate & 2.450 & 50 & 1.25 & 0.840 & 1.05 & 0.95 & 0.35 \\
Minimum rate & 2.650 & 0.5 & 1.25 & 0.768 & 0.95 & 1.05 & 0.65 \\
\hline
\end{tabular}

multi-component plasma (MCP) of ion species $j=1,2, \ldots$, with atomic numbers $A_{j}$ and charge numbers $Z_{j}$. The number density $n_{j}$ of ions $j$ can be expressed through the mass density $\rho$ of the matter as $n_{j}=X_{j} \rho /\left(A_{j} m_{\mathbf{u}}\right)$, where $X_{j}$ is the mass fraction of ions $j$ and $m_{\mathbf{u}}=1.66055 \times 10^{-24} \mathrm{~g}$ is the atomic mass unit. In a not too dense matter, almost all the mass is contained in the nuclei; the total mass fraction contained in the nuclei is $X_{N}=\sum_{j} X_{j} \approx 1$. At densities higher than the neutron drip density $\left(\sim 4 \times 10^{11} \mathrm{~g} \mathrm{~cm}^{-3}\right)$ free neutrons appear and $X_{N}<1$. Let us also define the fractional number $x_{j}=n_{j} / n$ of nuclei $j$ among other nuclei, with $\sum_{j} x_{j}=1$ and $n=\sum_{j} n_{j}$. Using these definitions, the mean charge and mass number of ions can be expressed as $\langle Z\rangle=\sum_{j} x_{j} Z_{j}$ and $\langle A\rangle=\sum_{j} x_{j} A_{j}$, respectively.

In a previous work [2] we propose a simple phenomenological formula for a reaction rate among equal nuclei, which combines all five nuclear buming regimes. A generalization of this expression to MCP was reported in Ref. [3]. According to [3] the rate of a reaction involving nuclei $i$ and $j$ can be written as

$$
R_{i j}(\rho, T)=R_{i j}^{\mathrm{pyc}}(\rho)+\Delta R_{i j}(\rho, T),
$$

where $R_{i j}^{\mathrm{pyc}}(\rho)$ is the zero-temperature pycnonuclear reaction rate and $\Delta R_{i j}(T)$ accounts for the temperature-dependent part. In the zero-temperature pycnonuclear regime the Coulomb barrier is penetrated owing to zero-point vibrations of ions around their equilibrium positions in a Coulomb crystal. For numerical evaluations, we have [3]

$$
R_{i j}^{\mathrm{pyc}}=10^{46} C_{\mathrm{pyc}} \frac{8 \rho X_{N} x_{i} x_{j} A_{i} A_{j}\langle A\rangle Z_{i}^{2} Z_{j}^{2}}{\left(1+\delta_{i j}\right)\left(A_{i}+A_{j}\right)^{2}} S\left(E_{i j}^{\mathrm{pk}}\right) \widetilde{\lambda}_{i j}^{3-C_{\mathrm{pl}}} \exp \left(-\frac{C_{\mathrm{exp}}}{\left(\widetilde{\lambda}_{i j}\right)^{1 / 2}}\right) \mathrm{cm}^{-3} \mathrm{~s}^{-1},
$$

where $\delta_{i j}$ is the Kronecker delta, which excludes double counting of the same collisions in reactions with identical nuclei. The constants $C_{\text {pyc }}, C_{\mathrm{pl}}$ and $C_{\text {exp }}$ are given in Table 2 (from Ref. [3]). The optimal model seems to be most reliable. Maximum rate and minimum rate models give the upper and the lower limits of the reaction rates, respectively [3]. The main parameter regulating the reaction rate is $\widetilde{\lambda}_{i j}=\alpha_{\lambda} \lambda_{i j}$, with $\alpha_{\lambda}$ given in Table 2 and

$$
\lambda_{i j}=\frac{A_{i}+A_{j}}{A_{i} A_{j} Z_{i} Z_{j}\left(Z_{i}^{1 / 3}+Z_{j}^{1 / 3}\right)}\left(\frac{\rho X_{N}\langle Z\rangle}{\langle A\rangle 1.3574 \times 10^{11} \mathrm{~g} \mathrm{~cm}^{-3}}\right)^{1 / 3} .
$$


The temperature-dependent part of the reaction rate is

$$
\Delta R_{i j}(\rho, T)=\frac{n_{i} n_{j}}{1+\delta_{i j}} \frac{S\left(E_{i j}^{\mathrm{pk}}\right)}{\hbar} r_{\mathrm{B} i j} P F,
$$

where $r_{\mathrm{B} i j}=\hbar^{2} /\left(2 \mu Z_{i} Z_{j} e^{2}\right)$. In Eq. (10), $F$ is an exponential function and $P$ is a preexponent term. According to Ref. [3],

$$
F=\exp \left(-\widetilde{\tau}_{i j}+C_{i j}^{\mathrm{sc}} \widetilde{\Gamma}_{i j} \varphi \mathrm{e}^{-\Lambda \widetilde{T}_{i j}^{(p)} / T}-\Lambda \frac{\widetilde{T}_{i j}^{(p)}}{T}\right)
$$

with

$$
\begin{aligned}
& \widetilde{\tau}_{i j}=3\left(\frac{\pi}{2}\right)^{2 / 3}\left(\frac{E_{a}}{k_{\mathrm{B}} \widetilde{T}}\right)^{1 / 3}, \quad \widetilde{\Gamma}_{i j}=\frac{Z_{i} Z_{j} e^{2}}{a_{i j} k_{\mathrm{B}} \widetilde{T}}, \quad \widetilde{T}=\sqrt{T^{2}+C_{T}^{2}\left(T_{i j}^{(p)}\right)^{2}}, \\
& T_{i j}^{(p)} \equiv \frac{\hbar \omega_{i j}}{k_{\mathrm{B}}}, \quad \widetilde{T}_{i j}^{(p)} \equiv \frac{\hbar \widetilde{\omega}_{i j}}{k_{\mathrm{B}}}, \quad \widetilde{\omega}_{i j}=\alpha_{\omega} \omega_{i j}, \quad E_{a}=\frac{2 \mu Z_{i}^{2} Z_{j}^{2} e^{4}}{\hbar^{2}} \\
& a_{i j}=\frac{a_{i}+a_{j}}{2}, \quad a_{i}=\left[3 Z_{i} /\left(4 \pi n_{e}\right)\right]^{1 / 3}, \quad \omega_{i j}^{2}=4 \pi Z_{i} Z_{j} e^{2} n_{i j} /(2 \mu),
\end{aligned}
$$

where $n_{i j}=3 /\left(4 \pi a_{i j}^{3}\right)$ and $n_{e}$ is the electron number density. The parameters $C_{T}$ and $\alpha_{\omega}$ are listed in Table 2. In Eq. (11), $\varphi=\sqrt{\Gamma_{i j}} /\left[\left(C_{i j}^{\mathrm{sc}} / \zeta_{i j}\right)^{4}+\Gamma_{i j}^{2}\right]^{1 / 4}, \Gamma_{i j}=Z_{i} Z_{j} e^{2} /\left(a_{i j} k_{\mathrm{B}} T\right)$ and $C_{i j}^{\mathrm{sc}}$ is associated with the strongly screened thermonuclear burning,

$$
\begin{aligned}
& C_{i j}^{\mathrm{sc}}=0.9\left[\left(Z_{i}+Z_{j}\right)^{5 / 3}-Z_{i}^{5 / 3}-Z_{j}^{5 / 3}\right] \frac{Z_{i}^{1 / 3}+Z_{j}^{1 / 3}}{2 Z_{i} Z_{j}}, \\
& \zeta_{i j}=\left(\frac{3\left\langle Z^{2}\right\rangle\left(Z_{i}^{1 / 3}+Z_{j}^{1 / 3}\right)^{3}}{8\langle Z\rangle Z_{i} Z_{j}}\right)^{1 / 2} .
\end{aligned}
$$

The quantity $\widetilde{T}$ is a renormalized temperature. It reflects the fact that the thermal energy $k_{\mathrm{B}} T$ of plasma ions in the thermonuclear regime is replaced by a temperatureindependent energy of zero-point ion motion in the pycnonuclear regime. The preexponent term can be written as

$$
P=\frac{8 \pi^{1 / 3}}{\sqrt{3} 2^{1 / 3}}\left(\frac{E_{a}}{k_{\mathrm{B}} \widetilde{T}}\right)^{\gamma} .
$$

The quantity $\gamma$ and the reaction energy $E_{i j}^{\mathrm{pk}}$ in the argument of the astrophysical factor $S\left(E_{i j}^{\mathrm{pk}}\right)$ can be chosen as [3],

$$
\gamma=\left(T^{2} \gamma_{1}+\left(\widetilde{T}_{i j}^{(p)}\right)^{2} \gamma_{2}\right) /\left(T^{2}+\left(\widetilde{T}_{i j}^{(p)}\right)^{2}\right)
$$




$$
E_{i j}^{\mathrm{pk}}=\hbar \widetilde{\omega}_{i j}^{(p)}+\left(\frac{Z_{i} Z_{j} e^{2}}{a_{i j}}+\frac{k_{\mathrm{B}} T \tau_{i j}}{3}\right) \exp \left(-\frac{\Lambda \widetilde{T}_{i j}^{(p)}}{T}\right),
$$

where $\gamma_{1}=2 / 3, \gamma_{2}=(2 / 3)\left(C_{\mathrm{pl}}+0.5\right), \tau_{i j}=3(\pi / 2)^{2 / 3}\left(E_{a} / k_{\mathrm{B}} T\right)^{1 / 3}$, and $\Lambda$ is given in Table 2 .

Finally, we note that the thermonuclear reaction regime with weak plasma screening is realized at rather high $T$ and low $\rho$, at which $\Gamma_{i j}<<1$. In this case the plasma effects on the reaction rate are minor while in other regimes they are huge. As temperature decreases and density increases, the thermonuclear regime with strong screening takes place, as long as $T>T_{i j}^{(p)}$. The intermediate thermo-pycnonuclear regime occurs in the temperature range $T_{i j}^{(p)} / 2<T<T_{i j}^{(p)}$. At even lower temperatures, $T>0.5 T_{i j}^{(p)} / \ln \left[Z_{i} Z_{j} e^{2} /\left(a_{i j} k_{\mathrm{B}} T_{i j}\right)\right]$, and higher densities, thermally enhanced pycnonuclear burning regime takes place. At lower $T$ the pycnonuclear burning rate becomes temperature independent, although it strongly depends on density.

The solution of the Coulomb tunneling (plasma physics) problem presented in this section seems sufficiently accurate at $T>T_{i j}^{(p)}$. At lower $T$ the reaction rate becomes rather uncertain and, in addition, depends on (generally unknown) microphysical structure of MCP (a regular lattice, or a lattice of microcrystals, or a lattice with impurities and defects, or a uniform mix). The presented equations are best suited for a uniform $\operatorname{mix}[3]$.

Our unified phenomenological expression presented here can be used, for instance, to determine the carbon ignition curve in a MCP environment, a crucial ingredient for modeling carbon explosions of massive white dwarfs as type Ia supernovae and nuclear explosions in surface layers of accreting neutron stars. Pycnonuclear effects in carbon ignition become important at $T<2 \times 10^{8} \mathrm{~K}$ and $\rho>10^{9} \mathrm{~g} \mathrm{~cm}^{-3}$.

\section{CONCLUSIONS}

The low energy astrophysical $S$-factors are most important for calculating fusion reaction rates in dense stellar matter. We have used the SP potential on the basis of the BP model to calculate $S(E)$ for the ${ }^{12} \mathrm{C}+{ }^{12} \mathrm{C},{ }^{12} \mathrm{C}+{ }^{16} \mathrm{O}$, and ${ }^{16} \mathrm{O}+{ }^{16} \mathrm{O}$ reactions.

We have presented a unified phenomenological equation for the reaction rate valid for all five burning regimes. The expression contains several parameters which can be varied to account for current theoretical uncertainties of the reaction rates.

\section{ACKNOWLEDGMENTS}

This work was partially supported by The Joint Institute for Nuclear Astrophysics (JINA) NSF PHY 0216783 and by the Russian Foundation for Basic Research (grants 05-02-16245, 05-02-22003). 


\section{REFERENCES}

1. L. R. Gasques, L. C. Chamon, D. Pereira, M. A. G. Alvarez, E. S. Rossi Jr., C. P. Silva and B. V. Carlson, Phys. Rev. C69, 034603 (2004).

2. L. R. Gasques, A. V. Afanasjev, E. F. Aguilera, M. Beard, L. C. Chamon, P. Ring, M. Wiescher, and D. G. Yakovlev, Phys. Rev. C72, 025806 (2005).

3. D. G. Yakovlev, L. R. Gasques, A. V. Afanasjev, M. Beard, and M. Wiescher, Phys. Rev. C74, 035803 (2006).

4. E. E. Salpeter and H. M. Van Horn, Astrophys. J. 155, 183 (1969).

5. L. C. Chamon et al., Phys. Rev. C66, 014610 (2002).

6. L. R. Gasques, L. C. Chamon, C. P. Silva, D. Pereira, M. A. G. Alvarez, E. S. Rossi Jr,, V. P. Likhachev, B. V. Carlson, and C. De Conti, Phys. Rev. C65, 044314 (2002).

7. E. S. Rossi Jr., D. Pereira, L. C. Chamon, C. P. Silva, M. A. G. Alvarez, L. R. Gasques, J. Lubian, B. V. Carlson, and C. De Conti, Nucl. Phys. A707, 325 (2002).

8. J. R. Patterson, H. Winkler, and C. S. Zaidins, Astrophys. J. 157, 367 (1969).

9. M. G. Mazarakis and W. E. Stephens, Phys. Rev. C7, 1280 (1973).

10. M. D. High and B. Cujec, Nucl. Phys. A282, 181 (1977).

11. K. U. Kettner, H. Lorenz-Wirzba, and C. Rolfs, Z. Phys. A298, 65 (1980),

12. H. W. Becker, K. U. Kettner, C. Rolfs, and H. P. Trautvetter, Z. Phys. A303, 305 (1981).

13. K. A. Erb and D. A. Bromley, Phys. Rev. C23, 2781 (1981).

14. B. Dasmahapatra, B. Cujec, and F. Lahlou, Nucl. Phys. A384, 257 (1982).

15. L. J. Satkowiak, P. A. DeYoung, J. J. Kolata, and M. A. Xapsos, Phys. Rev, C26, 2027 (1982).

16. P. Rosales et al., Rev. Mex. Fís. 49, 88 (2003).

17. E. F. Aguilera et al., Phys. Rev. C73, 064601 (2006).

18. L. Barrón-Palos et al., Nucl. Phys. A779, 318 (2006).

19. T. Spillane et al., Phys. Rev. Lett. 98, 122501.

20. B. Cujec and C. A. Bames, Nucl. Phys. A266, 461 (1976).

21. P. R. Christensen, Z. E. Switkowskiw, and R. A. Dayras, Nucl. Phys. A280, 189 (1977).

22. H. Spinka and H. Winkler, Nucl, Phys. A233, 456 (1974).

23. D. G. Kovar et al., Phys. Rev. C20, 1305 (1979).

24. G. Hulke, C. Rolfs, and H.-P. Trautvetter, Z. Physik A297, 161 (1980).

25. S.-C. Wu and C. A. Barnes, Nucl. Phys. A422, 373 (1984).

26. J. Thomas, Y. T. Chen, S. Hinds, D. Meredith, and M. Olson, Phys. Rev. C33, 1679 (1986).

27. A. Kuronen, J. Keinonen, and P. Tikkanen, Phys. Rev. C35, 591 (1987). 